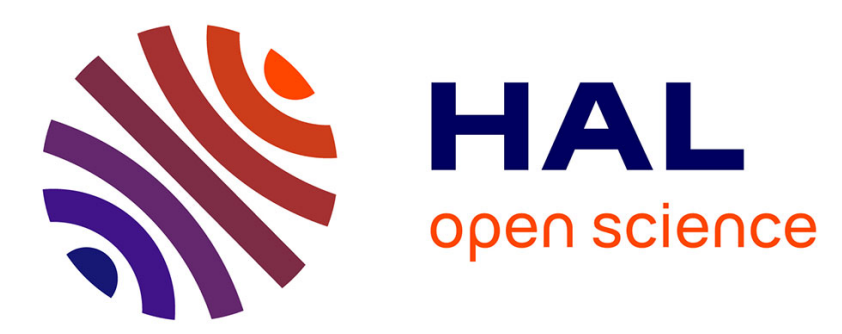

\title{
Neural-based Underwater Spherical Target Localization through Electrolocation
}

\author{
Yannick Morel, Vincent Lebastard, Frédéric Boyer
}

\section{To cite this version:}

Yannick Morel, Vincent Lebastard, Frédéric Boyer. Neural-based Underwater Spherical Target Localization through Electrolocation. 2015 IEEE International Conference on Robotics and Automation (ICRA), 2015, Washington State Convention Center, United States. 10.1109/ICRA.2015.7138975 . hal-01503239

\section{HAL Id: hal-01503239 \\ https://hal.science/hal-01503239}

Submitted on 6 Apr 2017

HAL is a multi-disciplinary open access archive for the deposit and dissemination of scientific research documents, whether they are published or not. The documents may come from teaching and research institutions in France or abroad, or from public or private research centers.
L'archive ouverte pluridisciplinaire HAL, est destinée au dépôt et à la diffusion de documents scientifiques de niveau recherche, publiés ou non, émanant des établissements d'enseignement et de recherche français ou étrangers, des laboratoires publics ou privés. 


\title{
Neural-based Underwater Spherical Target Localization through Electrolocation
}

\author{
Yannick Morel, Vincent Lebastard, and Frédéric Boyer
}

\begin{abstract}
Navigation of cluttered underwater environments remains to this day a challenging task in mobile robotics. Applying an electric field to a mobile robot's direct environment and measuring perturbations of this field, one is able to detect the presence of obstacles in close proximity of the system. In addition, one is also able to infer a range of information relative to the detected objects, such as their position or electrical characteristics. Extracting such information from available measures typically requires a model (analytical, numerical or heuristic) descriptive of the relationship from geometry of the scene to measures performed (typically referred to as forward model), or of the inverse relationship (inverse model). In the following, we directly extract one such model from experimental data, and capture a forward model using a neural formalism. Then, using an iterative procedure, we are able to estimate the position of a detected object and assess the degree of confidence one can place on this estimate. Merit of the approach is illustrated using experimental data for a spherical obstacle.
\end{abstract}

\section{INTRODUCTION}

Autonomous or remotely controlled robotic operations in unstructured, cluttered environments have long been recognized as constituting a significant challenge ([1]). The situation is to a large extent due to difficulties in using acoustic methods, such as SOund Navigation And Ranging (SONAR), in such a setting, as the clutter leads to severe multipath issues (see, among many others, [2] for a discussion of the manner in which multipath affects SONAR). Operational problems are only compounded in the case of murky, muddy, or turbid water, in which conditions vision is of little assistance in perceiving the environment. As a result, a wide range of applicative situations, in which robotics could provide invaluable support, have remained beyond the capabilities of existing technology, such as for instance in active environmental monitoring for rivers, ponds, and lakes, exploration of underwater wrecks, or search and rescue missions in capsizing ships.

To overcome such limitations, roboticists have over the years investigated novel sensing modalities. Of particular interest in the aforementioned setting is the electric sense ( [3]-[5]). This active sensing modality, exploited by a number of fish species, is based on the manipulation of electric fields. Specifically, the fish generates one such field and continuously measures deformations of this field using electroreceptor located along its body. The presence of objects (inert or biotic), whose electric properties differ from those of the surrounding fluid, will perturb the field. Based on the information collected, the fish is typically able to infer detailed representations of its immediate surroundings, including the presence, location, and general geometry of foreign objects (obstacles), or of living organisms (prey).
This sensing modality is characterized by a fairly short range (for the fish, typically a one-body length detection bubble around its body), however it has the advantages of being omnidirectional, well-suited to cluttered environments, and able to operate in turbid water. For these reasons, a number of research groups have investigated the application of a similar sensing principle to robotics (see for instance [6], [7]).

The typical functionality pursued has been that of underwater obstacle detection and localization (referred to hereafter as electro-location). Similarities between the considered problem and that investigated in Electrical Impedance Tomography (EIT, [8]) have motivated the use of EIT methods (see [9]). However, the number of electrodes (and therefore of available measures) on electro-location sensors is fairly limited. As a result, the inverse problem (of estimating the impedance map in the sensor's surroundings as a function of electric measures performed) is generally ill-posed ([10]). To reduce the dimension of the solution being estimated, it has in some instances been assumed that, in the sensor's direct environment, a significant portion of the volume is occupied by the surrounding fluid, whereas only a discrete number of obstacles are present. Therefore, the problem can be simplified by estimating the position of these obstacles, rather than attempting to construct a detailed impedance map of the environment. Such approaches have been very successful, with in particular techniques based on probabilistic estimation methods ([11]), or stochastic approaches ([12]).

In the following, we pursue a similar approach, in which we attempt to reconstruct the position of a given obstacle, present in the sensor's environment. The methods builds upon the technique used in [13], [14] to estimate relative positions in a group of robotic swimmers using the electric sense. In a first step, a forward map, from relative position of an obstacle with respect to the sensor to the electric measures performed by the sensor, is constructed. This forward map can assume a variety of forms, such as an analytical model (as that in [7] or in [15]), or a numerical model (e.g. based on Boundary Element Methods, BEM, [16], [17], such as that in [18]). In the following, we use experiments to collect data descriptive of this forward map. Then, we train a polynomial neural network ([19], [20]) to learn this forward map. In a second step, when attempting to localize an obstacle, series of candidate (or prospective) obstacle positions are presented as inputs to the trained neural net. The computed neural outputs (which correspond to predicted electric measures for each considered candidate positions) are then compared with the actual electric measures. The comparison allows to discern likelier positions of the obstacle. 
The main contribution of the paper lies in presenting a novel electro-location technique for obstacle localization, technique which had to this day only been applied to the relative position estimation problems for two mobile systems equipped with the electric sense (or, equivalently, to two electric sensors). One of the main strengths of the approach lies in its expected generalizability. In particular, the problem of developing analytic models describing the effect of the presence of simple (in a geometrical sense, e.g. spherical) obstacles on electric measures has been addressed (see [7], [21]). However, that same problem remains open for more complex object geometries, be it for localized obstacles of arbitrary shape, or continuous, non-homogeneously curved walls. The corresponding analytical derivations are likely to be rapidly become untractable, and computing numerical BEM models of arbitrarily detailed scenes can prove prohibitively time-consuming. Yet, the electric sense is, to a large extent, a rather myopic sense. Specifically, if the presence of an obstacle can be discerned from available measures at a given range, distance to the object must be reduced to close-to-zero to distinguish geometrical features. For example, presence of a cube or of a sphere produces electric measures which are essentially identical at a reasonable range (see [22]). In other words, from far enough, most objects look alike from an electric point of view. Accordingly, one could expect to be able to successfully address a large number of localization problems (i.e. localization for a large class of different objects) by exploring similarities between the electric measures corresponding to a number of generic shapes. This library of measures could be constructed using analytical or numerical models. However, following the approach used hereafter and collecting data experimentally instead, one is able to circumvent issues stemming from complexity of derivation or computation, and achieve greater flexibility in terms of the types of shapes considered. Establishing one such library and using it to estimate geometry of a complex scene is however beyond the scope of the present paper. Instead, in the following, we show that the proposed approach can be used to localize a spherical obstacle using experimental data.

In Section II, we provide a rapid description of the experimental setup and compare collected measures to values obtained from an analytical model. Then, in Section III, we use a neural approach to capture the map going from relative position of the obstacle to electric measures. Inverting this forward map, we are able to localize a sphere as a function of performed electric measures, as discussed in Section IV. Results obtained using experimental data are provided. Section V concludes this paper.

\section{Experimental Setup And Collected Measures}

In later sections, we address the problem of localizing an object of interest, underwater, through manipulation of electric fields. The setting in which we have developed and

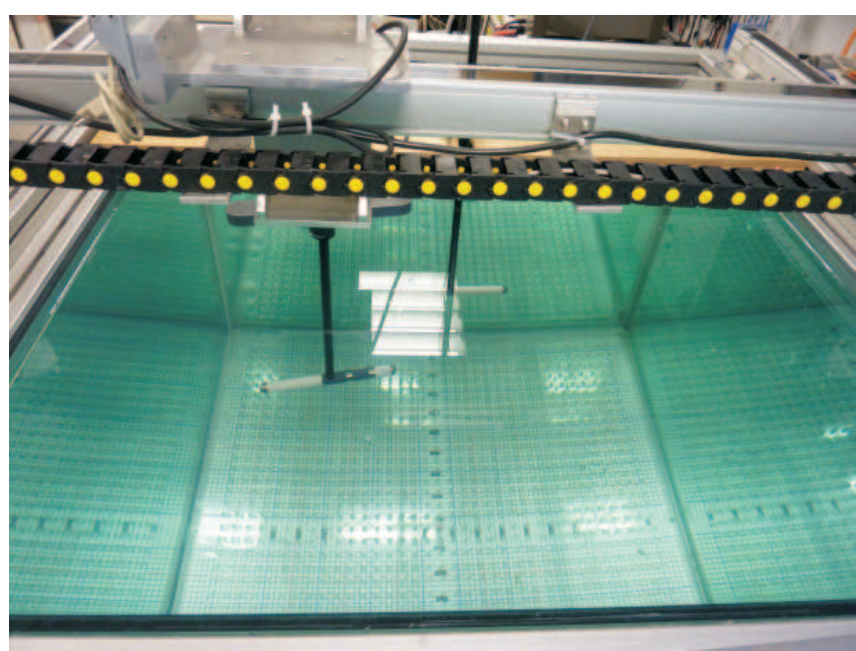

Fig. 1. Experimental setup, top down perspective. The system is shown with a pair of electric probes, visible as cylindrical objects in white and grey. The probe in the foreground is mounted on a three DoF actuation system, allowing to control its position and orientation in the horizontal plane.

tested the approach is described hereafter. At later stages of development, the technology is intended to equip mobile underwater vehicles. However, at this early developmental stage, the principles are tested on a dedicated experimental setup, shown in Figure 1.

\section{A. Experimental Setup}

The physical principle behind the object localization procedure is the following. A set of electrodes are used to generate an electric field and measure relevant electrical quantities. Specifically, in the setup used in the following, these electrodes are integrated within a slender cylinder (of length $21.6 \mathrm{~cm}$, diameter $2.5 \mathrm{~cm}$, probes seen in Figure 1 and Figure 2, see [7] for greater details). The electrodes are made of steel, the body of the cylinder itself is plastic and non-conductive. This cylinder is hereafter referred to as the probe or the electric probe. These probes are used within a $1 \mathrm{~m} \times 1 \mathrm{~m} \times 1 \mathrm{~m}$ glass tank, filled with fresh water. They are affixed at the extremity of a perch, rigidly connected to a three Degree of Freedom (DoF) actuation system, allowing movements in a horizontal plane (two translations, one rotation). A top-down view of the setup is provided in Figure 1. In the following, we will consider localization of a single object, a metallic sphere of radius $1 \mathrm{~cm}$.

The probe used features four pairs of electrodes. Two of these pairs form the probe's hemispheric extremities (see Figure 1 or Figure 2), the other two pairs are located on the fore part of the probe, their centers at $6.8 \mathrm{~cm}$ and $3 \mathrm{~cm}$ from the probe center, respectively. Each pair follows a strict leftright symmetry (see [7], [23]). A signal generator is used to apply a sinusoidal signal of frequency $22.5 \mathrm{KHz}$ to the aftmost hemispherical pair of electrodes. The (peak-to-peak) value of the current flowing through the three fore-most pairs, 


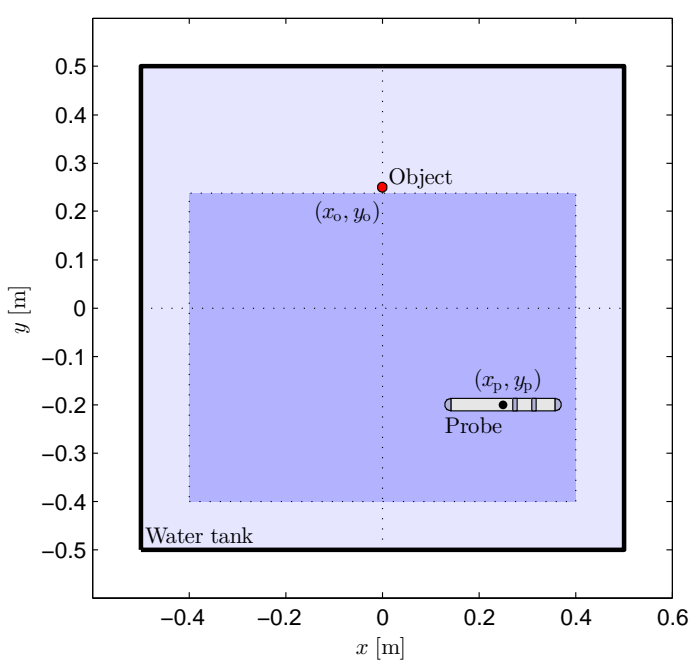

Fig. 2. Schematics of the experimental setup, the origin of the $x y$-frame of reference is placed at the center of the tank, a conductive (metallic) sphere of radius $1 \mathrm{~cm}$ is located at $\left(x_{\mathrm{o}}, y_{\mathrm{o}}\right)=(0 \mathrm{~m}, 0.25 \mathrm{~m})$, the position $(x, y)$ of the probe may span the area in a darker shade of blue.

which are connected to a common ground, is measured. For a more detailed description of the setup, the interested reader is referred to [23], [24].

Position of the obstacle and of the probe in the horizontal plane is described using an $x y$-frame of reference, the origin of which is located at the center of the setup (see Figure 2). To collect data to construct a forward map (from relative position to collected measures), we dispose the obstacle and probe as follows. The spherical obstacle remains fixed, its center located at $\left(x_{\mathrm{o}}, y_{\mathrm{o}}\right)=(0 \mathrm{~m}, 0.25 \mathrm{~m})$. The probe is free to move about the horizontal plane. However, for security purposes (in particular to prevent collisions with walls or the spherical obstacle), we will in general constrain its movement to the darker blue area in Figure 2; that is, to $(x, y) \in[-0.4 \mathrm{~m}, 0.4 \mathrm{~m}] \times[-0.4 \mathrm{~m}, 0.23 \mathrm{~m}]$. More specifically, to collect data the probe was made to travel along series of straight lines parallel to the $x$-axis in Figure 2, from $y=0 \mathrm{~m}$ to $y=0.225 \mathrm{~m}$ with $5 \mathrm{~mm}$ steps (further measures in the direction of $y$ decreasing are of limited use, as the effect of the obstacle's presence is not seen in the measures). For each such $y$-position, the probe is made to travel from $x=-0.37 \mathrm{~m}$ to $x=0.37 \mathrm{~m}$, with a speed of travel and a sample frequency such that we obtain a sample every $1.2 \mathrm{~mm}$ traveled in the $x$ direction. To isolate the contribution of the obstacle and eliminate influence of the walls, we repeated the same procedure without obstacle, and subtracted that reference value from the measures collected with the obstacle present. An example of the obtained measures is shown in Figure 3.

\section{B. Analytical Model}

For completeness and to offer additional insights into the manner in which presence of the obstacle impacts performed measures, we briefly present the analytical model of the currents measured, whose derivation is presented is detail in [7] (alternately, see [15] for fewer details and a higher level of abstraction). It is convenient to consider each pair of electrodes along the probe's body separately, and for any such pair to estimate (or measure) the total currents flowing through it, and the difference between the current flowing into the left electrode and that flowing into the right one. The former (the sum) produces signals of greater magnitude, which facilitates detection of a perturbation of the electric field. The latter (the lateral difference) provides more detailed information regarding the detected object's relative position with respect to the probe, in particular allowing to straightforwardly assess whether it is located port or starboard. For ease of presentation, we group the four current sums within $i_{\text {lo }} \in \mathbb{R}^{4}$, where lo stands for longitudinal and in which current sums are included from fore-most to aftmost pair. Similarly, we group the lateral current differences within $i_{\mathrm{la}} \in \mathbb{R}^{4}$ (la stands for lateral) in the same front-toback order. In practice, we are interested in the perturbation due to the presence of an obstacle. Accordingly, we typically subtract from $i_{\text {lo }}$ the values obtained in the absence of any obstacle (which serve as reference). That operation is not necessary for $i_{\mathrm{la}}$, due to the sensor's left-right symmetry. The value of $i_{\text {lo }}$ (due to the presence of a spherical obstacle) can be computed as follows ([7]),

$$
i_{\mathrm{lo}}=C_{0} K C_{0} u,
$$

where $C_{0} \in \mathbb{R}^{4 \times 4}$ is a conductance matrix whose expression depends on the probe's geometry, $u \in \mathbb{R}^{4}$ is the vector of electrical potentials applied to the four pairs of electrode (in our case $u=\left[\begin{array}{cccc}0 & 0 & 0 & u_{0}\end{array}\right]^{\mathrm{T}}$, where $u_{0}$ represents the signal applied to the aft-most electrode pair), and $K \in \mathbb{R}^{4 \times 4}$ is a matrix whose entries are of the form

$k_{i j}=-\frac{1}{4 \pi \gamma} \frac{r_{i}^{\mathrm{T}} \chi a^{3} r_{j}}{\left\|r_{i}\right\|^{3}\left\|r_{j}\right\|^{3}}, \quad i=1, \ldots, 4, j=1, \ldots, 4$,

where $k_{i j}$ is the $i^{\text {th }}$ line- $j^{\text {th }}$ column entry of $K, \gamma \in \mathbb{R}$ is the conductivity of the surrounding fluid, $a \in \mathbb{R}$ is the spherical obstacle's radius, $\chi \in \mathbb{R}$ its relative conductivity, and $r_{i} \in \mathbb{R}^{3}, i=1, \ldots, 4$, is the vector going from the obstacle's position to the center of the $i^{\text {th }}$ pair of electrodes.

Similarly, the $i^{\text {th }}$ entry of $i_{\text {la }}$ can be computed as follows,

$$
\begin{array}{r}
i_{\mathrm{la} i}=S_{\perp} \frac{\gamma \chi a^{3} \bar{A}_{i}}{\left\|r_{i}\right\|^{5}}\left(2\left(n_{i}^{\mathrm{T}} r_{i}\right) r_{i}+\left(n_{i}^{\mathrm{T}} r_{i \perp}\right) r_{i \perp}\right) E_{0}, \\
i=1, \ldots, 4,
\end{array}
$$

where $S_{\perp} \in \mathbb{R}$ is a scaling factor, $\bar{A}_{i} \in \mathbb{R}$ characterizes the surface of electrode pair $i, n_{i} \in \mathbb{R}^{3}$ is a vector characterizing the direction of the surface of the $i^{\text {th }}$ electrode pair, $r_{i \perp} \in \mathbb{R}^{3}$ is a vector of same magnitude as and perpendicular to $r_{i}$, and $E_{0} \in \mathbb{R}^{3}$ is the electric field produced by the sensor, evaluated at the position of the obstacle, which can be 


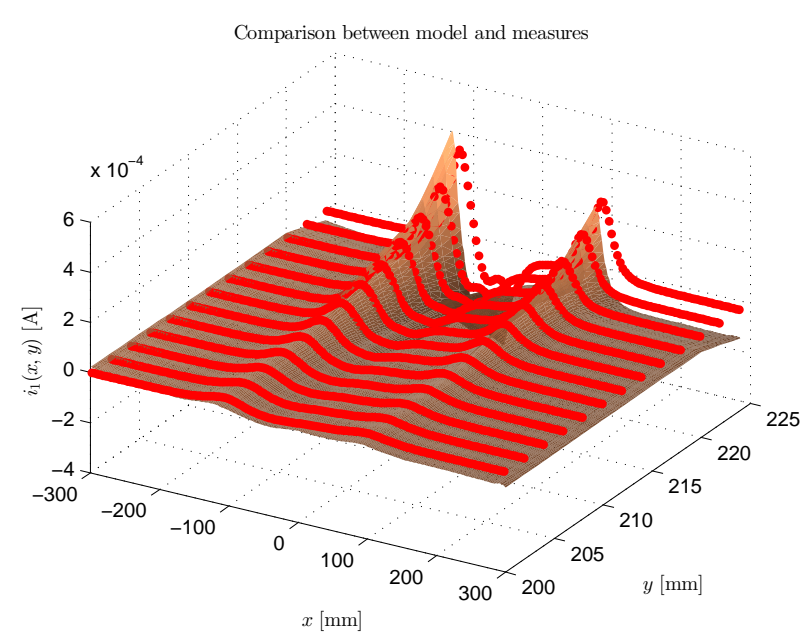

Fig. 3. Comparison between experimental measures (represented using a linearly interpolated surface) and model predictions (marked with red dots).

computed as

$$
E_{0}=\frac{1}{4 \pi \gamma} \sum_{i=1}^{4} \frac{C_{0} u_{i}}{\left\|r_{i}\right\|^{3}} r_{i}
$$

where $u_{i}$ is the $i^{\text {th }}$ entry of vector $u$.

We used the model given by (1)-(4) to compute the currents expected in a configuration identical to the one for which we collected experimental data. Experimental measures (shown as a linearly interpolated surface) are compared to model values (represented with red dots) in Figure 3. Represented is the first component of $i_{\text {lo }}$, with the position of the obstacle kept constant at $\left(x_{\mathrm{o}}, y_{\mathrm{o}}\right)=(0 \mathrm{~m}, 0.25 \mathrm{~m})$, and values of current shown for the sensor traveling over $(x, y) \in[-0.3 \mathrm{~m}, 0.3 \mathrm{~m}] \times[-0.20 \mathrm{~m}, 0.225 \mathrm{~m}]$. The current is represented on the $z$-axis as a function of the $x y$-position of the probe.We note that the exact position of the peaks is slightly different between model and experiment, and the background current value (away from the peaks) is not as uniform on the experimental data as it is in the case of the analytical model. The latter is certainly to a large extent due to imperfect compensation of the walls' influence on experimental measures. Nevertheless, we note that general trends are a good match, which tends to confirm that measures are representative of the phenomenon investigated, as described by the above analytical model.

\section{FORWARD NEURAL MODEL}

The experimental data collected, as discussed in the previous section, is descriptive of the forward map $f(\cdot)$ going from relative position of the sensor with respect to that of the obstacle, to the current measures obtained for that relative position,

$$
\begin{aligned}
f: \mathcal{D}_{x} \times \mathcal{D}_{y} & \rightarrow \mathcal{D}_{i}, \\
x, y & \rightarrow i,
\end{aligned}
$$

where $\mathcal{D}_{x}, \mathcal{D}_{y} \subset \mathbb{R}, \mathcal{D}_{i} \subset \mathbb{R}^{6}, x, y$, represent the position of the probe in the frame of reference shown in Figure 2 (position which, assuming the obstacle remains fixed at $\left(x_{\mathrm{o}}, y_{\mathrm{o}}\right)$, is representative of the sensor's relative position with respect to the obstacle), and $i$ represents the vector of useful current measures obtained for a sensor position of $(x, y)$. Note that the analytical model's formulation leads, for the type of probe considered (featuring four pairs of electrodes) to eight different current measures available. However, measures obtained on the fourth pair are typically a linear combination of that obtained from the other electrode and are thus non-informative. In the measure vector $i(x, y)$, we therefore only include the first three values in $i_{\mathrm{lo}}(x, y)$ and $i_{\text {la }}(x, y)$ (corresponding to values obtained from the foremost three pairs of electrodes).

Following the approach proposed in [13], [14], we use a neural formalism to capture the input-output map (5) as described by the experimental data collected. Specifically, from the form of model (1)-(4), we note that so long as the obstacle's position differs from that of the center of any electrode pair (which in practice is always verified), then $f(x, y)$ as given in (5) is a continuous function of its arguments. Hence, from Weierstrass' approximation theorem ([25]), we can conclude that for any $\varepsilon>0$ there exists a polynomial function $\hat{f}(x, y)$ on $\mathcal{D}_{x} \times D_{y}$ such that

$$
\|f(x, y)-\hat{f}(x, y)\|<\varepsilon, \quad(x, y) \in \mathcal{D}_{x} \times D_{y} .
$$

Hereafter, we use the following structure for $\hat{f}(x, y)$ (which corresponds to that of a Single Hidden Layer Neural Network, SHL-NN, [26]),

$$
\hat{f}(x, y) \triangleq \varphi(x, y) W, \quad(x, y) \in \mathcal{D}_{x} \times \mathcal{D}_{y},
$$

where we choose $\varphi(x, y) \in \mathbb{R}^{p}$ as a row vector of polynomial functions of $x, y$, and $W \in \mathbb{R}^{p \times 6}$ is a matrix of neural weights to be selected. The learning (of the forward map (5)) task is accomplished by selecting $W$ in such a manner that (7) becomes descriptive of (5) and of the experimental data collected. A wide range of methods can be used to achieve that goal. Specifically, numbering the experimental measures discussed in the previous section from 1 to $n$, let $X \triangleq\left[\begin{array}{lll}x_{1} & \ldots & x_{n}\end{array}\right]^{\mathrm{T}} \in$ $\mathbb{R}^{n}, Y \triangleq\left[\begin{array}{lll}y_{1} & \ldots & y_{n}\end{array}\right]^{\mathrm{T}} \in \mathbb{R}^{n}, I(X, Y) \triangleq$ $\left[i_{1}^{\mathrm{T}}=f^{\mathrm{T}}\left(x_{1}, y_{1}\right) \quad \ldots \quad i_{n}^{\mathrm{T}}=f^{\mathrm{T}}\left(x_{n}, y_{n}\right)\right]^{\mathrm{T}} \in \mathbb{R}^{n \times 6}$, and define

$\Phi(X, Y) \triangleq\left[\begin{array}{lll}\varphi^{\mathrm{T}}\left(x_{1}, y_{1}\right) & \ldots & \varphi^{\mathrm{T}}\left(x_{n}, y_{n}\right)\end{array}\right]^{\mathrm{T}} \in \mathbb{R}^{n \times p}$.

Then consider

$$
W^{*} \triangleq \Phi^{\dagger}(X, Y) I(X, Y)
$$

where ${ }^{\dagger}$ denotes the usual pseudo-inverse. The particular choice of weights $W=W^{*}$ minimizes in the Least Mean Square (LMS) sense the reconstruction error $f\left(x_{i}, y_{i}\right)-$ $\hat{f}\left(x_{i}, y_{i}\right), i=1, \ldots, n$. That choice of weights is used in 
the following. Note that, alternately, one could use Bayesian inference in the parameter space to estimate the maximum (a posteriori) likelihood $W$ matrix, as a function of available measures (as in [27]). Such an approach presents the advantage of allowing to explicitly account for measurement noise when estimating $W$. However, in the case considered here, comparisons show that the accuracy of the obtained forward map is not noticeably improved over the LMS solution.

\section{INVERSION OF THE FORWARD MODEL: LOCALIZATION}

Inverting the forward model identified in the previous section can be accomplished by considering the distance between different sample points in the measurement space $\mathcal{D}_{i}$. Specifically, for a given measure $i(t)$, performed at time instant $t \geqslant 0$, consider the reconstruction error

$$
e\left(i(t), \eta_{\mathrm{c}}\right) \triangleq\left\|i(t)-\hat{f}\left(\eta_{\mathrm{c}}\right)\right\|, \quad t \geqslant 0
$$

where $\eta_{\mathrm{c}} \triangleq\left[\begin{array}{ll}x_{\mathrm{c}} & y_{\mathrm{c}}\end{array}\right]^{\mathrm{T}} \in \mathcal{D}_{x} \times \mathcal{D}_{y}$ represents a candidate position of the sensor. Then, consider the relative position estimate chosen as

$$
\hat{\eta}(t) \triangleq \underset{\eta_{\mathrm{c}} \in \mathcal{D}_{x} \times \mathcal{D}_{y}}{\operatorname{argmin}} e\left(i(t), \eta_{\mathrm{c}}\right), \quad t \geqslant 0,
$$

where $\hat{\eta} \triangleq\left[\begin{array}{ll}\hat{x} & \hat{y}\end{array}\right]^{\mathrm{T}}, \eta \triangleq\left[\begin{array}{ll}x & y\end{array}\right]^{\mathrm{T}} \in \mathcal{D}_{x} \times \mathcal{D}_{y}$, are the estimated and actual positions, respectively. It can be shown (see [13]) that, assuming the inverse map is sufficiently smooth, the more accurate our neural model (i.e. the smaller $\varepsilon$ in (6)), and the more closely $\hat{f}(\hat{\eta})$ matches the actual measure $i$, the closer our estimate $\hat{\eta}$ is to the actual relative position $\eta$.

In practice, the approach only works if the sensor is within detection range of the object; that is, if it is close enough that the perturbation to the electric field due to the object's presence is important enough to be registered (which depends on measurement noise levels, magnitude of the electric field produced by the sensor, and geometry of the object). When outside of this detection range, the object's presence has no influence on measures. The approach however, generalizes elegantly to such cases. In particular, to exploit the above insights to perform localization, we use grids of candidate positions. Computing the reconstruction error (9) over the entire grid, it becomes straightforward to assess likelier areas. Specifically, in the case that the information measured is sufficiently informative (i.e. if close enough), the approach yields results that explicitly discriminate the most likely candidate position (for which the error is minimal), from unlikely candidates (with high error). In the case that the measured information is not sufficient, the ambiguity is made apparent by the results. Typically, large swaths appear as being good possible candidates. In that manner, considering how specifically the method is discriminating a likelier candidate position, it becomes possible to not only estimate the obstacle's position, but also assess the degree of confidence we can have in the estimate produced. The results can be represented in a manner that is visually intuitive. In particular, considering the contour plot of the reconstruction error over an area of interest, assessing likely and unlikely positions can be done by distinguishing areas of low reconstruction errors from areas of higher reconstruction error. One such contour plot is provided in Figure 4.

In the case considered, a metallic sphere of radius $1 \mathrm{~cm}$ is present in position $\left(x_{\mathrm{o}}, y_{\mathrm{o}}\right)=(0 \mathrm{~m}, 0.25 \mathrm{~m})$. The probe is made to travel on a straight line, parallel to the $x$-axis. The probe's actual trajectory is shown in green (with initial and final positions marked with an $\times$ and a , respectively). As the probe moves along the green trajectory, samples of $i_{\mathrm{lo}}$ and $i_{\text {la }}$ are measured. Using the forward map discussed in Section III and the above inversion procedure, we estimate the position of the probe with respect to that of the obstacle. These estimates are marked with $\times$ 's in Figure 4. They were obtained using an iterative procedure. Specifically, for each sample we began with a candidate grid spanning $(x, y) \in$ $[-0.3 \mathrm{~m}, 0.3 \mathrm{~m}] \times[0 \mathrm{~m}, 0.22 \mathrm{~m}]$ with steps of $10 \mathrm{~mm}$ in the $x$-direction and $5 \mathrm{~mm}$ in $y$. Then, we used a second grid, centered on the most likely candidate of the previous grid (chosen according to (10)), with a grid width of $60 \mathrm{~mm}$ in $x$ and $y$, and $1 \mathrm{~mm}$ steps. A third gird is then used, centered on the estimate obtained from the second grid, with widths of $10 \mathrm{~mm}$ in $x$ and $y$, and $0.1 \mathrm{~mm}$ steps. We note that the obtained estimates are reasonably faithful, as they remain close to the actual trajectory. The contour levels shown in Figure 4 are those of the reconstruction error (9) for the final position shown (marked with a $\cdot$ ). The lower the error (dark blue), the more similar the reconstructed measures are to the available set of measures. The greater the error (bright red), the more dissimilar. Accordingly, positions appearing in dark blue are likely candidate positions, while positions appearing in other colors (from green, to yellow and red) are unlikely. This information can be exploited in different manners. In particular, the candidate of least error represents in some sense the most likely candidate position (marked with $\times$ 's). However, in cases that there are vast areas of low error (such as is the case in Figure 4, with a swath of low values at about $x=100 \mathrm{~mm}$, from $y=0 \mathrm{~mm}$ to $200 \mathrm{~mm}$, approximately), the specific position of this minimum is not necessarily very informative, and it is useful to consider the gradient of the error (or slope of the corresponding error surface, if representing $e$ as a surface over $x-y$ ). Both aspects can be combined using a Luenberger-form estimator ([28]). In black, we show the result obtained following one such approach. The model used is limited to kinematics and is descriptive of the fact that the probe is traveling in a direction parallel to the $x$-axis, at an a priori known speed (of $5 \mathrm{~mm} / \mathrm{s}$ ). Within this model, we included system noise on speed and heading (additive Gaussian noises, with means 0deg and $0.1 \mathrm{~mm} / \mathrm{s}$, standard deviations $20 \mathrm{deg}$ and $0.75 \mathrm{~mm} / \mathrm{s}$ ) to reflect movement uncertainty. In addition, we exploited 


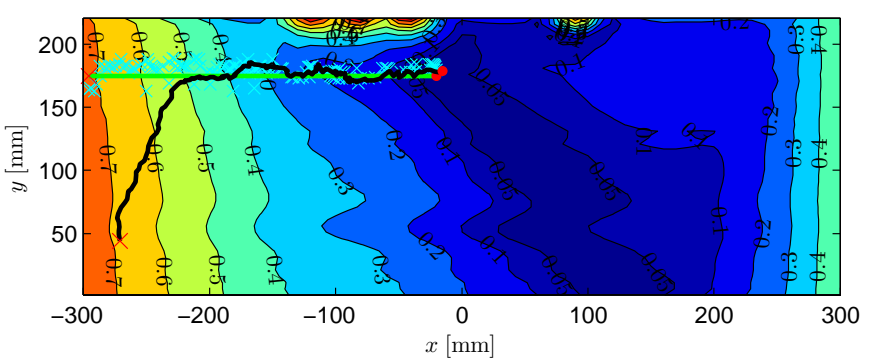

Fig. 4. Level curves of the reconstruction error $e(x, y)$, indicating likelier (blue, low error) and less likely (in red, high error) positions of the probe; actual probe trajectory in green, instantaneous estimates marked with cyan $\times$ 's, Luenberger observer trajectory in black.

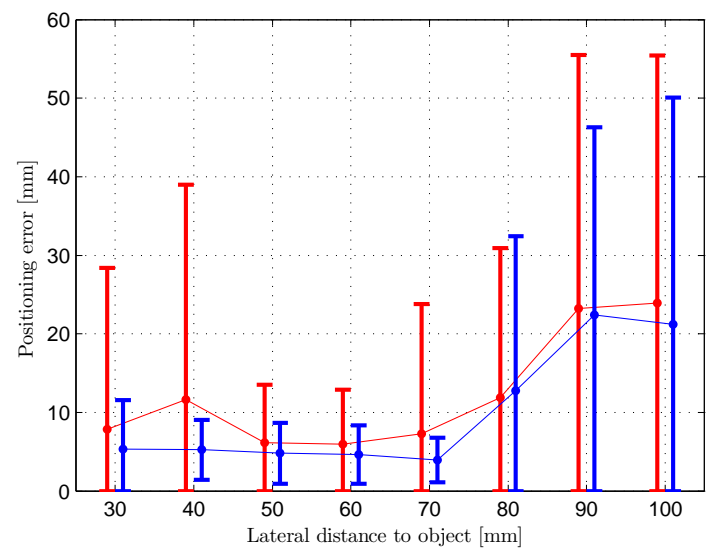

Fig. 5. Localization error for increasing lateral distances of the obstacle, means marked with dots, standard deviations represented with brackets; in red, values obtained with the probe traveling from $x=-300 \mathrm{~mm}$ to $300 \mathrm{~mm}$, in blue, with the probe traveling from $x=-300 \mathrm{~mm}$ to $0 \mathrm{~mm}$.

the information inferred from electric measures to design corrective terms. A first term is chosen proportional to the difference between current $x y$-position of the Luenberger observer and that of the position of least reconstruction error. A second term (akin to a derivative term in a Proportional Derivative controller) is chosen proportional to the gradient of $e(x, y)$ evaluated at the $x y$-position of the observer. While the probe travels along the line $y=175 \mathrm{~mm}$ with an initial $x$-position at $x=-296 \mathrm{~mm}$, the initial condition of the observer is chosen as $(x, y)=(-270 \mathrm{~mm}, 40 \mathrm{~mm})$, to illustrate convergence of the estimate produced to the actual trajectory of the probe. As seen in Figure 4, the information is sufficiently accurate for the observed trajectory (in black) to converge to a neighborhood of the actual one (in green).

Finally, note that, as previously discussed, the closer the probe is to the obstacle, the greater the amplitude of the measures and the easier the estimation of the position. To quantify the impact of range, we made the probe travel in straight lines parallel to the $x$-axis (from $x=-300 \mathrm{~mm}$ to $300 \mathrm{~mm}$ ), and increasing lateral distance progressively, starting at $y=220 \mathrm{~mm}$ (lateral distance of $30 \mathrm{~mm}$ from the obstacle), up to $y=150 \mathrm{~mm}$ (lateral distance of $100 \mathrm{~mm}$ ). The mean positioning error for each trajectory is represented with a in Figure 5, and the standard deviation is represented with a bracket (in red). Note that, as seen for instance in Figure 2, the probe is dissymmetric, with three pairs of electrode on the fore part, and only one located aft. The idea behind that specific design choice is that, if the probe figuratively represents a mobile vehicle, it is more interested in discerning what lies ahead (in the probable direction of movement) than what is behind. As a result, as the probe travels from $x=-300 \mathrm{~mm}$ to $x=300 \mathrm{~mm}$, information collected (and therefore localization), is significantly better when the obstacle (situated in $x=0 \mathrm{~mm}$ ) lies in front of the probe. To verify this, we show in blue in Figure 5 the same error means and standard deviations, but only accounting for travel from $x=-300 \mathrm{~mm}$ to $x=0 \mathrm{~mm}$. As expected, the localization errors are noticeably smaller.

\section{CONCLUSiON}

The work presented concerns the localization of spherical objects using electrolocation; that is, through the process of applying an electric field to the sensor's direct environment, measuring perturbations of this field due to the presence of foreign objects, and inferring information relative to these objects based on measured information. The approach uses experimental data to capture, using a SHL-NN, the forward map from obstacle relative position to the corresponding expected electric measures. Then, when detecting the presence of an obstacle and attempting to reconstruct its position, the neural forward map is inverted using an iterative procedure. A series of grids of candidate positions are presented to the neural map, which outputs the corresponding expected current measures. Comparing measures associated to different candidate positions to the actual measure performed allows to discern likelier candidate positions. We show, using experimental data, that the approach allows to estimate the obstacle position when within range, with a mean positioning error that is function of the distance between sensor and obstacle (the closer the sensor, the better the estimate). The main contribution of the paper lies in presenting a new solution to this electrolocation problem. In addition, this solution presents a number of interesting features when compared to existing alternate techniques. In particular, the approach is readily generalizable to any particular shape of obstacle, provided the corresponding data can be collected (to build the forward map). Conversely, typical methods rely on either analytical or numerical models, which, for complex geometries may become either untractable or require prohibitive computational efforts. In addition, the approach provides a clear map of space around the sensor, which allows to intuitively discern (a) what is the obstacle's likeliest position (if any), (b) what degree of confidence one may place on the estimate, and (c) if the estimate is uncertain, what areas of space are likelier to be occupied and which ones are likelier to be free. In the future, the approach will be extended to consider scenes composed of several 
objects. Specifically, the approach presented here will be supplemented by a neural classifier ([29]) and rely on a library of captured neural models corresponding to different generic objects' forward maps (such as the one used here, which corresponds to spheres of a given radius). When presented with a new scene, in a first stage, the approach will use the neural classifier to classify the type of scene perceived (in terms of number and type of objects). Then, the localization approach proposed here will exploit the available library of forward maps to compose an estimate of the scene under consideration.

\section{REFERENCES}

[1] C. Thorpe and H. Durrant-Whyte, "Field robots," in Proc. of the $10^{\text {th }}$ Int. Symp. of Robotics Research, (Lorne, Australia), 2001.

[2] W. C. Knight, R. G. Pridham, and S. M. Kay, "Digital signal processing for SONAR," Proc. of the IEEE, vol. 69, no. 11, pp. 1451-1506, 1981.

[3] H. W. Lissmann and K. E. Machin, "The mechanism of object location in Gymnarchus niloticus and similar fish," Journ. of Experimental Biology, vol. 35, no. 2, pp. 451-486, 1958.

[4] W. Heiligenberg and J. Bastian, "The electric sense of weakly electric fish," Annual review of physiology, vol. 46, no. 1, pp. 561-583, 1984.

[5] G. von der Emde, M. Amey, J. Engelmann, S. Fetz, C. Folde, M. Hollmann, M. Metzen, and R. Pusch, "Active electrolocation in Gnathonemus petersii: Behaviour, sensory performance, and receptor systems," Journal of Physiology-Paris, vol. 102, no. 4, pp. 279290, 2008.

[6] M. A. McIver and M. E. Nelson, "Towards a biorobotic electrosensory system," Autnomous Robots, vol. 11, pp. 263-266, 2001.

[7] F. Boyer, P.-B. Gossiaux, B. Jawad, V. Lebastard, and M. Porez, "Model for a sensor inspired by electric fish," IEEE Trans. on Robotics, vol. 28, no. 2, pp. 492-505, 2012.

[8] M. Cheney, D. Isaacson, and J. C. Newell, "Electrical impedance tomography," SIAM review, vol. 41, no. 1, pp. 85-101, 1999.

[9] J. Snyder, Y. Silverman, Y. Bai, and M. A. MacIver, "Underwater object tracking using electrical impedance tomography," in 2012 IEEE/RSJ Int. Conf. on Intelligent Robots and Systems (IROS), pp. 520-525, 2012.

[10] A. P. Calderón, "On an inverse boundary value problem," Comp. Appl. Math, vol. 25, no. 2-3, 2006.

[11] J. R. Solberg, K. M. Lynch, and M. A. McIver, "Active electrolocation for underwater target localization," The International Journal on Robotic Research, vol. 27, no. 5, pp. 529-548, 2008.

[12] V. Lebastard, C. Chevallereau, A. Girin, N. Servagent, P.-B. Gossiaux, and F. Boyer, "Environment reconstruction and navigation with electric sense based on a kalman filter,' Int. Journ. of Robotics Research, vol. 32, no. 2, pp. 172-188, 2013.

[13] Y. Morel, M. Porez, and A. J. Ijspeert, "Estimation of relative position and coordination of mobile underwater robotic platforms through electric sensing," in Proc.
2012 IEEE Int. Conf. on Rob. and Aut., (St. Paul, MN), pp. 1131-1136, 2012.

[14] Y. Morel, M. Porez, and A. J. Ijspeert, "Actionperception trade-offs for anguilliform swimming robotic platforms with an electric sense," in Proc. 2012 IFAC Workshop on Navigation, Guidance and Contr. of Underwater Vehicles, (Porto, Portugal), 2012.

[15] F. Boyer and V. Lebastard, "Exploration of objects by an underwater robot with electric sense," in Biomimetic and Biohybrid Systems, pp. 50-61, 2012.

[16] P. K. Banerjee, The Boundary Element Methods in Engineering. McGraw-Hill College, 1994.

[17] L. C. Wrobel and M. H. Aliabadi, The Boundary Element Method. New Jersey: Wiley \& Sons, 2002.

[18] M. Porez, V. Lebastard, A. J. Ijspeert, and F. Boyer, "Multi-physics model of an electric fish-like robot: $\mathrm{Nu}$ merical aspects and application to obstacle avoidance," in Proc. 2011 IEEE/RSJ Int. Conf. on Intelligent Robots and Systems, (San Francisco, CA), 2011.

[19] Y. Shin and J. Ghosh, "Approximation of multivariate functions using ridge polynomial networks," in Proc. IEEE Int. Joint Conf. on Neural Networks 1992, vol. 2, (Baltimore, MD), pp. 380-385, 1992.

[20] C. K. Chak, G. Feng, and C. M. Cheng, "Orthogonal polynomials neural network for function approximation and system modeling," in Proc. IEEE Int. Joint Conf. on Neural Networks 1992, vol. 1, (Beijing, China), pp. 594-599, 1995.

[21] B. Rasnow, "The effect of simple objects on the electric field of Apteronotus," Journal of Comparative Physiology, vol. 178, no. 3, pp. 397-411, 1996.

[22] V. Lebastard, C. Chevallereau, A. Girin, F. Boyer, and P.-B. Gossiaux, "Localization of small objects with electric sense based on Kalman filter," in IEEE Int. Conf. on Robotics and Automation (ICRA), pp. 11371142, 2012.

[23] V. Lebastard, C. Chevallereau, A. Girin, F. Boyer, and P. B. Gossiaux, "Localization of small objects with electric sense based on kalman filter," in Proc. 2012 IEEE Int. Conf. on Rob. and Aut., (St. Paul, MN), pp. 1137-1142, 2012.

[24] F. Boyer, P. B. Gossiaux, B. Jawad, V. Lebastard, and M. Porez, "Model for a sensor inspired by electric fish," IEEE Transactions on Robotics, vol. 52, no. 2, pp. 492505, 2012.

[25] H. Jeffreys and B. S. Jeffreys, Methods of Mathematical Physics, 3rd Ed. Cambridge, England: Cambridge University Press, 2000.

[26] J. T. Spooner, M. Maggiore, R. Ordòñez, and K. M. Passino, Stable Adaptive Control and Estimation for Nonlinear Systems. New York, NY: John Wiley and Sons, Inc, 2002.

[27] C. E. Rasmussen, Gaussian Processes for Machine Learning. Cambridge, MA: MIT Press, 2006.

[28] D. G. Luenberger, "Observer for multivariable systems," IEEE Trans. on Automatic Control, vol. AC-11, no. 2, pp. 190-197, 1966.

[29] S. Haykin, Neural Networks: A Comprehensive Foundation. Upper Saddle River, NJ: Prentice Hall, 2004. 